\title{
Comparison between equivalent spring element method and additional mass method in nuclear power structure
}

\author{
Jin-Xiao Wang ${ }^{1, a}$, Meng-Yan Song ${ }^{2, a}$, Peng Fu, ${ }^{3, a}$ \\ Fen $\mathrm{He}^{4, \mathrm{~b}^{*}}$ \\ ${ }^{1}$ Department of Civil Engineering, China Agricultural University, Beijing, 100083 China \\ ${ }^{2}$ China Nuclear Power Engineering Co, Ltd, Beijing, 100840, China \\ ${ }^{3}$ Cabr Construction Consulting Co, Ltd, 100013, China \\ ${ }^{4}$ Chinese Academy of Agricultural Engineering, 100125, China \\ awangzhx@163.com, bhefen_2005@163.com
}

Keywords: Equivalent spring element method; Additional mass method; Dynamic characteristics Abstract. In order to study the effect of fluid on the structural dynamic characteristics, in this paper, a three-dimensional spring element was applied to the dynamic analysis of the fluid. Based on the simplified model of nuclear power structure, the upper water tank of concrete containment was analyzed in different water level by equivalent spring element method and additional mass method. The theory of three-dimensional equivalent spring element was put forward, and then the numerical simulation of different water tank was carried out. The computational results of the two methods showed that the equivalent spring element method and the additional mass method have the similar accuracy.

\section{Introduction}

For the third generation of nuclear power structuret, the test or numerical simulation of nuclear power structure can almost not be found from the literature, as the third generation of reactor type at the top of the fluid solid coupling of the storage tank, how to simulate the analysis is less. Yu Xiaoyu ${ }^{[1]}$ and Dang Junjie ${ }^{[2]}$ used additional mass method in analysis of nuclear power containment cooling water storage tank structure, through experiments and other numerical examples demonstrate the feasibility and effectiveness of additional mass method. Ying Xiumei ${ }^{[3]}$ applied the simplified theory of the rigid body spring element to the double layer graphite granular structure, and gave the theoretical basis of the stiffness and damping equivalent of spring element. The spring element was proposed by Professor Kawai ${ }^{[4]}$ in Japan firstly. The traditional finite element method can handle the continuous medium. This paper compared the equivalent spring element method with the additional mass method and the feasibility of the spring element method for the numerical simulation of nuclear power structure was studied.

\section{Formula derivation of spring element}

The three prism of any cross section can be equivalent to a three-dimensional spring element model. In Fig. 1, the three section length prism are a, b, c, the height is $h$. The formula for calculating the equivalent spring element is as follows: The cross bar area formula:

$$
A_{1-2}=A_{4-5}=\frac{A}{6} \cdot \frac{b}{\gamma h} \text { (1) } \quad A_{2-3}=A_{5-6}=\frac{A}{6} \cdot \frac{c}{\gamma h} \text { (2) } \quad A_{1-3}=A_{4-6}=\frac{A}{6} \cdot \frac{a}{\gamma h}
$$

The Oblique bar area formula:

$$
\begin{gathered}
A_{1-5}=A_{2-4}=\frac{A}{6} \cdot \frac{b^{2}+h^{2}}{\left(h^{2}-\gamma b^{2}\right)} \cdot \frac{\sqrt{b^{2}+h^{2}}}{h} \\
A_{3-5}=A_{2-6}=\frac{A}{6} \cdot \frac{c^{2}+h^{2}}{\left(h^{2}-\gamma c^{2}\right)} \cdot \frac{\sqrt{c^{2}+h^{2}}}{h}
\end{gathered}
$$




$$
A_{1-6}=A_{3-4}=\frac{A}{6} \cdot \frac{a^{2}+h^{2}}{\left(h^{2}-\gamma a^{2}\right)} \cdot \frac{\sqrt{a^{2}+h^{2}}}{h}
$$

The spring rigidity formula:

$$
K=\frac{E A}{L}
$$

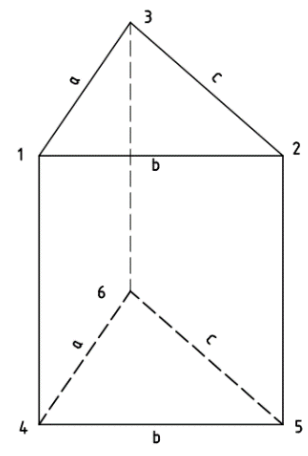

Fig.1 Solid model

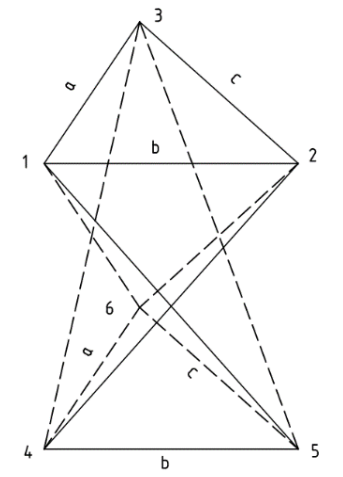

Fig.2 Equivalent spring element model

\section{The illustration of nuclear power structure}

\section{The size of nuclear power structure}

The inner diameter of the shell is $48.57 \mathrm{~m}$, the thickness of concrete is $1.1 \mathrm{~m}$, the outer diameter of the water tank is $29.22 \mathrm{~m}$, the inner diameter is $9.76 \mathrm{~m}$, the water tank is $0.5 \mathrm{~m}$, the material is the same as the concrete shell.

\section{The material parameters of nuclear power structure}

Because theie is notdetailed parameters in the nuclear power structure, the concrete $\mathrm{C} 30$ is chosen as the material of the concrete shell. The bulk modulus of the water is $2.2 \mathrm{e} \mathrm{Pa}^{[5]}$.

\begin{tabular}{|c|c|c|c|}
\hline material & density $\left(\mathrm{kg} / \mathrm{m}^{3}\right)$ & Elastic(Pa) & Poisson's ratio \\
\hline concrete & 2400 & $3 \mathrm{e} 10$ & 0.2 \\
\hline & 1000 & $2.2 \mathrm{e} 9$ & 0.5 \\
\hline
\end{tabular}

Table 1 The parameters of each material

\section{Comparison between three kinds of quality method and spring element method}

The total water level in the tank is $6.088 \mathrm{~m}$. Calculate the height of the water level is $4 \mathrm{~m}, 5 \mathrm{~m}, 6 \mathrm{~m}$.

\section{Additional mass method}

In this paper, the density of the total mass of the water and the water tank in the water tank and the ratio of the volume of the water to the area are given to the water tank in the region to achieve the additional mass method. Such as the water in level $4 m$.

Table 2 Volume density in level $4 \mathrm{~m}$

\begin{tabular}{ccc} 
& \multicolumn{3}{c}{ Volume $\left(\mathrm{m}^{3}\right)$} & density $\left(\mathrm{kg} / \mathrm{m}^{3}\right)$ \\
\cline { 2 - 3 } water & 2109.532 & 1000 \\
concrete & 908.200 & 2400 \\
\hline
\end{tabular}

Equivalent density $\rho=2400+\frac{2109.532 \times 1000}{908.1997}=4722.762 \mathrm{~kg} / \mathrm{m}^{3}$

\section{Spring element method}

The arrangement of the spring element is shown in Fig. 3. The two particles are arranged along the vertical direction, and each layer has three concentric circles of $14.01 \mathrm{~m}, 9.67 \mathrm{~m}$ and $5.33 \mathrm{~m}$, respectively, along the circumferential direction of the 24 particles. 


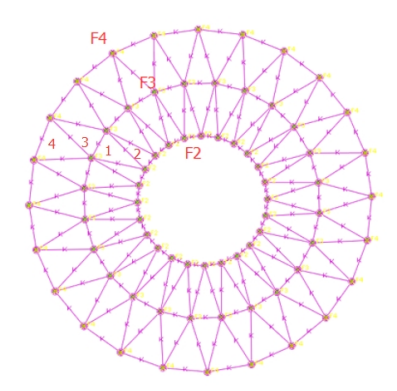

Fig.3 Spring element layout

\section{The comparison of first thirty order frequency}

The first 30 thirty order frequency of the two methods are as follows.

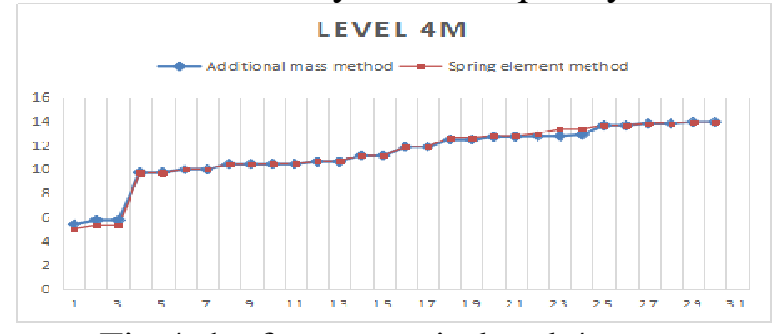

Fig.4 the frequency in level $4 \mathrm{~m}$

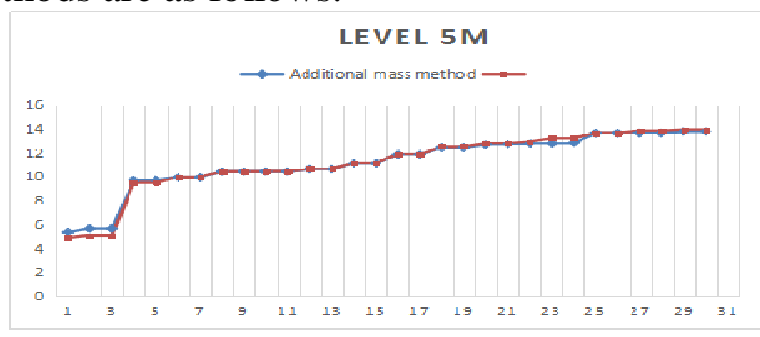

Fig. 5 the frequency in level $5 \mathrm{~m}$

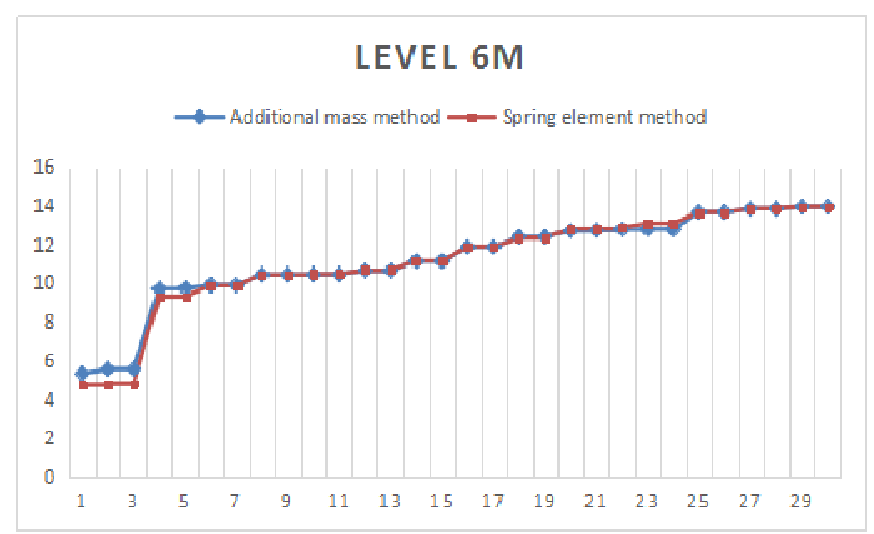

Fig. 6 the frequency in level $6 \mathrm{~m}$

\section{Conclusion}

This paper compares the different level, using added mass method and spring element method nuclear power structure concrete containment of the modal analysis results. A comparison of the two analysis method to obtain the order of formation and frequency is basically the same, both calculated formation diagram similar. In the engineering application,we found that the spring element can be used to simplify the fluid.

\section{Acknowledgements:}

Project supported by the National Natural Science Foundation of China (Nos. 11272340),, the Chinese Academy Of Agricultural Engineering(Nos. 201401).

Corresponding author Fen He, E-mail: hefen_2005@163.com

\section{References}

[1] Xiaoyu Yu. Study on added mass of pit filters in nuclear power plant. Shanghai Jiao Tong University, 2010 
[2] Junjie Dang. Under the action of earthquake of AP1000 passive containment cooling water storage box and a shield to build logistics solid coupled vibration characteristics of. North China Electric Power University, 2014.

[3] Jingjing Song. Application of rigid body spring element in granular structure: [Master Dissertation]. Beijing: Institute of water conservancy and civil engineering, China Agricultural University, 2013

[4] Kawai T. New element models in discrete structural analysis. Proceedings of the Japan Shipbuilding Institute. 1976, 141: 174-180.

[5] Zhenhua Lao,Zhaobo JiangThe experimental study on the relationship between the volume of liquid bulk modulus and temperature 
